\title{
Acute traumatic cervical spinal cord injury in a third-trimester pregnant female with good maternal and fetal outcome: a case report and literature review
}

\author{
Ashok Reddy Pedaballe ${ }^{1} \cdot$ Harvinder Singh Chhabra ${ }^{1} \cdot$ Vikas Tandon $^{1} \cdot$ Parashuram Chauhan $^{2} \cdot$ Rachna Verma $^{3}$
}

Received: 14 July 2018 / Revised: 30 September 2018 / Accepted: 7 October 2018

(c) International Spinal Cord Society 2018

\begin{abstract}
Background: The management of acute traumatic cervical spine injury in a third-trimester pregnancy is challenging with risks involved for both the mother and the fetus. We report one such case that was managed successfully with good maternal and fetal outcomes.

Case presentation: A 30 years female, gravida 2, para 1, living 1 at 31 weeks 5 days of pregnancy, met with a RTA and was diagnosed with AIS B C4-C5 extension compression spinal cord injury (SCI) with a viable fetus. Closed reduction of C4-C5 dislocation was achieved through controlled cervical traction. Having involved the patient in informed decision-making, anterior cervical discectomy and fusion (ACDF) was performed under general anesthesia (GA), with obstetrician, as well as neonatologist available in the operation theater. The pregnancy was uneventful in the post-operative stage. A healthy baby was delivered at 36 weeks of gestation through cesarean section. At final follow-up review of 12 months the patient was ambulatory without support and was able to perform most of the regular activities independently.

Discussion: The significant risk of a spontaneous delivery with GA posed the dilemma of either managing the injury conservatively through bed rest, continuing the pregnancy till its term and then opting for surgical stabilization after delivery or opting for surgical stabilization of the spine immediately, with a view for early mobilization and rehabilitation. A successful outcome of traumatic cervical SCI in third-trimester pregnancy can be achieved by multi-disciplinary (anesthetist, obstetrician, neonatologist, spine surgeon, and physiatrist) team, and timely surgical spinal stabilization, followed by early comprehensive rehabilitation.
\end{abstract}

\section{Introduction}

The incidence of traumatic spinal cord injury in a pregnant woman is quite low and only a few cases have been reported in the literature [1]. However, adequate literature is available for the management of pregnancy in women with spinal cord injuries [1]. The management of acute traumatic cervical spine injury in the third-trimester of pregnancy is

Ashok Reddy Pedaballe

drashokreddy@gmail.com

1 Spine Services, Indian Spinal Injuries Centre, Vasant Kunj Sector C, New Delhi, India

2 Department of Anaesthesia, Indian Spinal Injuries Centre, Vasant Kunj Sector C, New Delhi, India

3 Department of Obstetrics and Gynaecology, Indian Spinal Injuries Centre, Vasant Kunj Sector C, New Delhi, India challenging with regard to the risks involved for both the mother and the fetus and the difficulties in rehabilitation. We report one such case that was managed successfully with good outcomes for both the mother and fetus and review the literature for various aspects of the management.

\section{Case presentation}

A 30 -year-old female patient, gravida 2, para 1, living 1 at 31 weeks and 5 days of pregnancy met with a high-velocity RTA while traveling in a four-wheeler. She was given first aid, stabilized, and transferred to our tertiary care center $24 \mathrm{~h}$ later and kept under vasopressor (Inj. Dopamine) support. She was admitted to the intensive care unit and her vitals were stabilized. Comprehensive evaluation, including an MRI of the cervical spine and an ultrasonography (USG) of the abdomen were performed. She was diagnosed with AIS B C4-C5 extension compression SCI (Fig. 1) with a 


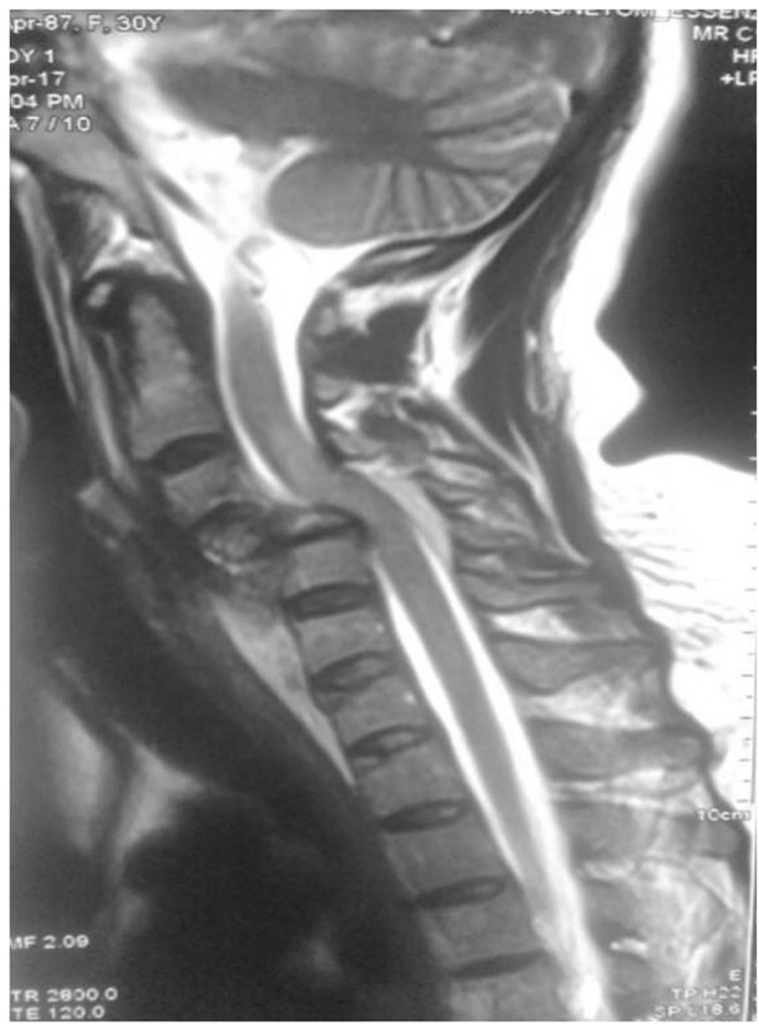

Fig. 1 MRI showing the extension compression injury C4-C5

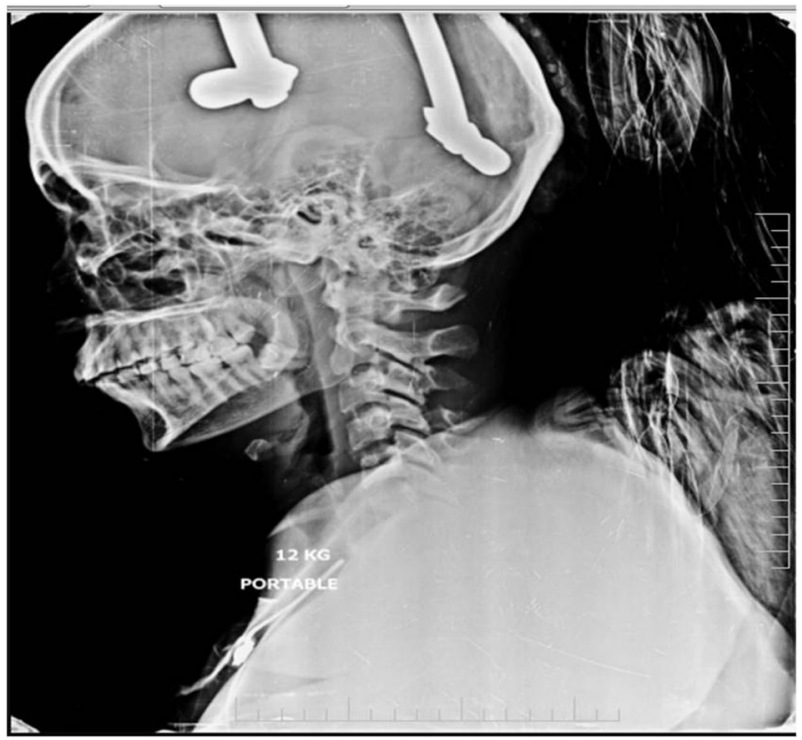

Fig. 2 Lateral radiograph with cervical traction

viable fetus, adequate liquor, and closed internal os. Controlled awake cervical skeletal traction was applied immediately and the C4-C5 dislocation was reduced by a sequential increase in weight to $12 \mathrm{~kg}$ (Fig. 2).

We were uncertain with regard to the further course of management. After the deliberations we narrowed down to

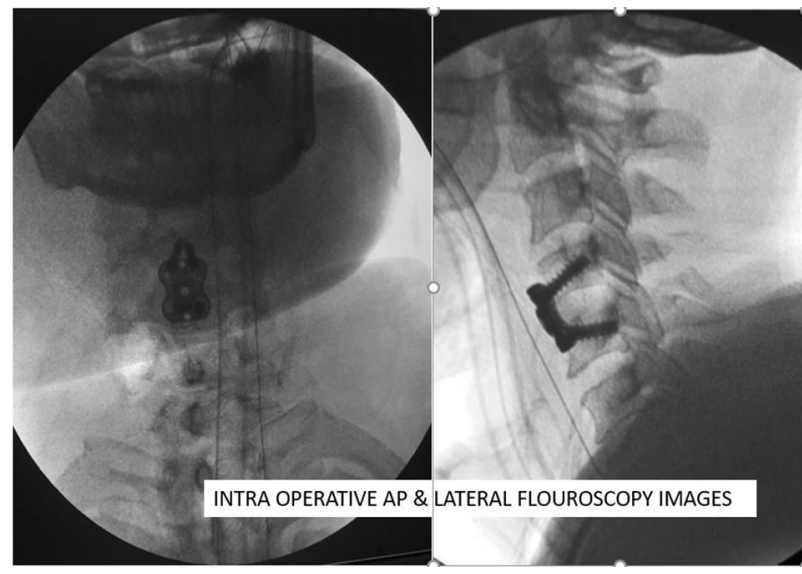

Fig. 3 Intraoperative fluoroscopy images of C4- C5 ACDF

two options: (i) managing the injury conservatively with bed rest, continuing the pregnancy till the end of its term and then opting for surgical stabilisation after delivery; or (ii) going in immediately for surgical stabilization of the spine with a view to early mobilisation and rehabilitation. An anesthetist, obstetrician, neonatologist, spine surgeon, and a physiatrist were involved in the decision-making as were the patient and attendants. ACDF C4-C5 was performed under GA, modifying the anesthetic agents, under the cover of tocolytics as well as continuous FHS monitoring and with an obstetrician, as well as a neonatologist available in the operation theater (Fig. 3). The duration of the radiation exposure was restricted, and the abdomen was shielded with lead aprons. The patient tolerated the surgery well and the immediate post-operative period was uneventful. Her rehabilitation began from the second postop day with minimal modifications of the protocol. As per the advice of the obstetrician and the patient's preference, her bladder was managed with an indwelling silicon catheter. The motor power of lower limbs improved gradually. At 36 weeks gestation, in view of respiratory discomfort and a previous history of delivery by cesarean section, an elective cesarean section was performed. She withstood the surgery well and a healthy baby girl was delivered (Table 1).

\section{Results}

The patient successfully underwent a cervical spine stabilization surgery. The pregnancy progressed till 36 weeks gestation when she underwent a cesarean section and delivered a healthy baby with 2.7 kilos of birthweight with an APGAR score of 10. There were no perioperative incidents. At a twelve-month follow up, the patient's neurological status had improved from AIS B to AIS D, her 
Table. 1 Time frame of events

\begin{tabular}{|c|c|}
\hline \multicolumn{2}{|l|}{ Time frame of Events } \\
\hline Within $24 \mathrm{~h}$ of the injury & Admitted to the tertiary care center \\
\hline Within $6 \mathrm{~h}$ of admission & Stabilization of the vitals, closed skeletal traction and reduction of the fracture. \\
\hline Next $48 \mathrm{~h}$ & $\begin{array}{l}\text { Two doses of Inj Beclomethasone } 12 \mathrm{mg} \mathrm{IM} \text { administered } 12 \mathrm{~h} \text { apart. Informed } \\
\text { decision-making }\end{array}$ \\
\hline After $72 \mathrm{~h}$ & C4-C5 ACDF under GA \\
\hline Second post-op day & Initiation of Rehabilitation \\
\hline Next 4 weeks & Continuation of rehabilitation \\
\hline At 36 weeks of gestation & Elective LSCS \\
\hline Next 2 weeks & Continuation of the rehabilitation as an in-patient \\
\hline $\begin{array}{l}\text { After a total } 6 \text { weeks of hospital } \\
\text { stay }\end{array}$ & Discharged \\
\hline Till 3 months & Continuation of rehabilitation and physiotherapy as domiciliary care \\
\hline At the $12^{\text {th }}$ month follow up & Independent ambulator, able to perform all ADL \\
\hline
\end{tabular}

Table. 2 Results

\begin{tabular}{lll}
\hline Results & \multicolumn{2}{l}{ Duration since time of injury } \\
\cline { 2 - 3 } Parameters & $\begin{array}{l}72 \mathrm{~h} \text {. post } \\
\text { injury }\end{array}$ & $\begin{array}{l}\text { At the } 12^{\text {th }} \text { month } \\
\text { follow up }\end{array}$ \\
\hline AMS (0-100) & 9 & 96 \\
ASS (0-112) & 112 & 112 \\
AIS grade (A-E) & B & D \\
SCIM score (100) & 15 & 95 \\
Self care (0-20) & 0 & 15 \\
Respiratory \& sphincter & 15 & 40 \\
management (0-40) & & 40 \\
Mobility score (0-40) & 0 & 20 level \\
WINSCI II questionnaire (Level & 0 level & \\
0-20) & & 230 \\
CUE -Q (34-238) & 34 & \\
\hline
\end{tabular}

American Spinal Injury Association(ASIA) Motor Score (AMS) had improved from 9 to 96, Capabilities of Upper Extremity (CUE) Score had improved from 36 to 230 (238 max), Spinal Cord Independence Measure (SCIM) score had improved from 15 to 95 , and the Walking index for spinal cord injury (WISCI-II) scale had improved from level 0 to level 20. She could perform all her routine activities independently (Table 2).

\section{Discussion}

\section{SCl during Pregnancy}

Though there is substantial literature on the management of pregnancy in an individual with a spinal cord injury, there is hardly any literature on the management of acute SCI in pregnant women. The few available case reports on managing SCI in pregnant women are mainly for non-traumatic SCI. When it comes to the management of cervical SCI in a pregnant woman, there is a paucity of literature [1, 2]. Hence, our dilemma about managing our situation.

Three case reports on managing SCI in pregnant women in their second trimester reported successful outcomes with early surgeries and continuation of the pregnancies [3-5]. One case series [6] with three patients of advanced pregnancy and SCI had poor fetal and maternal outcomes with conservative management. Emergency cesarean delivery and the surgical stabilization of the spine in this series resulted in improved neurology of the mothers, but the newborns had low birthweight and APGAR score (Table 3). Therefore, we opted for early surgical stabilization of the spine, early rehabilitation, and continuation of the pregnancy in this case.

Though emergency non-obstetric surgery can be done in any trimester, it is safest in the second trimester of pregnancy [7], when procedures under GA can be tolerated. In the third trimester, there are increased chances of spontaneous labor [8], and problems of a preterm delivery should be anticipated. Our patient was a 30 -year-old gravida 2, para 1, living 1 at 31 weeks and five days of gestation with $24 \mathrm{~h}$-history of AIS B acute traumatic cervical SCI, as a result of a $\mathrm{C} 4-\mathrm{C} 5$ fracture dislocation. It is now wellestablished that the multi-disciplinary approach plays a vital role in managing any SCI patient [9]. In a pregnant female patient with SCI, an obstetrician and a neonatologist also need to be included in the team. In this case, various modifications were done to the normal protocol of each discipline as per the needs of the pregnancy. The salient modifications are highlighted in the following text.

\section{Spine surgery}

As per dictum, when both the mother and the fetus are at risk due to a procedure, then the mother's health should be given priority [7]. In this case, the mother had an incomplete injury and had good chances of improvement in neurology with an early intervention. Hence, we 
successfully attempted an early reduction of the cervical dislocation with cervical traction. Once the reduction was achieved, the patient was given the options of: (i) continuing the pregnancy with bed rest till term/spontaneous delivery; or (ii) undergoing a spine fixation first, starting rehabilitation early, and continuing with the pregnancy till its term. Each strategy had its own advantages and disadvantages. With the first option, the advantages were that the fetus would not be exposed to any radiation and the risk of preterm delivery would not be increased. The disadvantages were that she would need to be on bed rest and thus, it would not be possible to start an early aggressive rehabilitation program. With the second option, the advantage of definitive stabilization of the spine and early commencement of rehabilitation would be there along with the disadvantage of exposure of the fetus to intraoperative radiation and risk of preterm delivery with general anesthesia. The patient was given an alternative option from her gynecologist elsewhere to first undergo an emergency cesarean section and plan the cervical spine surgery subsequently. However, we were not in favor of this strategy due to the disadvantage of a preterm delivery. After discussing the pros and cons of each strategy with the team of obstetrician, neonatologist, anesthetist, physiatrist, and spine surgeon, the patient and attendant chose the second option. Under continuous FHS monitoring, a C4-C5 ACDF was done. Grade IV and V Extension compression injuries usually require 360 degrees fusion [10], but in this case, only anterior fusion and stabilization was done, considering the risks of the prone position required for posterior stabilization. The fact that there might be a need for posterior surgery at a later date was explained to the patient.

\section{Thromboprophylaxis}

A pregnancy by itself is a hypercoagulable state with a 4-5 times higher risk of thromboembolism [11], and SCI further increases the risk of thromboembolism. Hence, thromboprophylaxis becomes very essential. We used both pharmacological and mechanical measures for thromboprophylaxis. Low molecular weight Heparin (LMWH) can be safely given during pregnancy for thromboprophylaxis [12]. Hence prophylactic injection Clexane $60 \mathrm{mg}$ was given subcutaneously over the thigh (not abdomen) once a day, and intermittent pneumatic compression device was used for both lower limbs.

\section{Radiographs}

Hazards of the exposure of the fetus to radiation in the third trimester are quite low. However, it should be avoided wherever possible [13]. The radiation exposure was minimized in this case through the following measures. 
i. Preoperatively: An MRI was done for the primary diagnosis and only two lateral view radiographs were done during the closed reduction by cervical traction. A CT scan was not taken.

ii. Intraoperatively: Localization of the level for the incision was done using surface marking of various anatomical landmarks. Use of fluoroscopy images was minimized to only the essential steps. Lead aprons were draped over the abdomen with four supports connected to OT table and the slings connected to the supports so that the weight of the aprons was not transferred to the abdomen (Fig. 4).

\section{Obstetric management}

Due to the risks of preterm delivery, an informed decision was taken by the patient and the attendants to continue the pregnancy till term or till any significant fetal/maternal distress was noticed under the vigilant protocol while giving her tocolytics [8]. Isoxsuprine [14], a beta-adrenergic drug, acts on the uterine smooth muscle and causes uterine relaxation (tocolysis). Isoxsuprine $10 \mathrm{mg}$ tablet was given every $6 \mathrm{~h}$, with regular FHS monitoring and periodic USG abdomen to look for any signs of fetal distress. To enhance the fetal lung maturity in anticipation of a preterm delivery, the administration of antenatal steroids (Beclomethasone or Dexamethasone) [15] is recommended. In this case, two doses of $12 \mathrm{mg}$ Inj. Betamethasone IM were given at a $24 \mathrm{~h}$ interval. Bladder training (CIC) was delayed and she continued with a Foley's indwelling catheter for two weeks, for reasons of physical comfort. At 36 weeks of gestation, the patient complained of respiratory discomfort and back pain, which began interfering with rehabilitation. Hence, she was given the option of an elective cesarean. Since the patient opted for an elective cesarean, it was performed and a healthy baby was delivered. The postpartum period was uneventful.

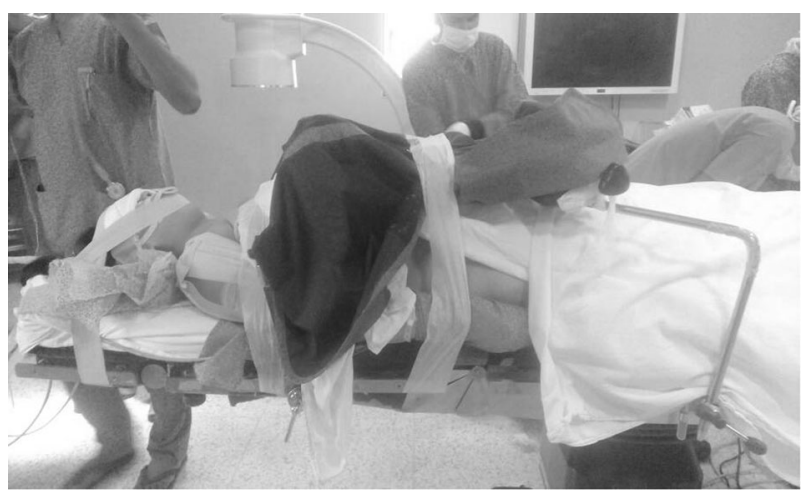

Fig. 4 Positioning and use of lead apron as a shield over the abdomen with side supports

\section{Anesthesia}

At this stage of pregnancy ( 31 weeks and 5 days), there is a high risk of spontaneous delivery during the perioperative period with GA [8]. This must be explained to the patient and informed consent should be taken if surgery is planned. Tocolytics should be given perioperatively [7] to avoid premature uterine contraction and spontaneous delivery. Inj. Isoxsuprine $10 \mathrm{mg}$ slow IV stat was given before shifting the patient to the operation theater and the infusion was continued during the administration of anesthesia.. However, the infusion was withheld due to hypotension. Postoperatively, Inj. Isoxsuprine $10 \mathrm{mg}$ IV bolus was given and repeated every $6 \mathrm{~h}$, for $24 \mathrm{~h}$, every $8 \mathrm{~h}$ for the next $24 \mathrm{~h}$, and then continued for five days with a $10 \mathrm{mg}$ tablet three times a day.

\section{Positioning}

The risk of aortocaval compression by the uterus is more in the supine position. This can cause supine hypotension which can be effectively avoided in the left lateral decubitus or wedging [7]. Since the anterior cervical spine surgery had to be performed in the supine position, we applied the wedge under the right hip, prophylactically. However, the patient had supine hypotension and was able to maintain blood pressure only with inotropic (Dopamine) support.

\section{Anaesthetics}

Propofol was used for induction and Rocuronium bromide for intubation. The anesthesia was maintained with oxygen, air, isoflurane, and propofol mixed with ketamine (5:1 ratio), so as to avoid a fall in the BP. Nitrous oxide was avoided since it increases blood flow to the brain (by decreasing the vascular resistance) and increases the risk of intracranial hemorrhage in the premature fetus $[8,16]$. The patient was reversed with neostigmine and glycopyrrolate. After reversal, the patient was extubated.

After 1 month (at 36 weeks of pregnancy), elective cesarean section (for obstetric reasons) was performed under routine general anesthesia. Sedatives were avoided until the delivery of the fetus.

\section{Rehabilitation}

Rehabilitation of pregnant women with SCI is challenging due to the difficulties in mobilization, transfers, skin integrity issues, bladder and bowel care, and the association of complications like autonomic dysreflexia (if neurological level proximal to T6), deep venous thrombosis and UTI [17]. The weight and increased skin integrity issues due to pregnancy necessitated greater vigilance and skin care. The 
increased weight and the protuberant abdomen posed additional difficulty in transfers and mobilisation, leading to greater dependence even after she had regained reasonable motor power. Standing on an "Oswestry frame" required a slight modification and tilt table was not used in order to avoid pressure over the abdomen.

Bladder Care: The protuberant abdomen in the third trimester can pose difficulties in doing a self-clean intermittent catheterisation (CIC). Our patient was reluctant to perform CIC, therefore an indwelling silicon catheter was utilised for two weeks. The risks of UTI are higher during pregnancy, and as recommended by the literature, she was prescribed a prophylactic dose of Nitrofurantoin, an oral bacteriostatic drug for asymptomatic bacteriuria [18]. After two weeks her bladder-control improved, and she was able to self-void.

Bowel care: Constipation is a frequent problem with spinal injuries, and pregnancy compounds the issue [19]. Our patient initially had difficulty in regular bowel movement. However, the problem resolved with a more rigorous implementation of bowel management program.

\section{Indian scenario}

This case was managed in a center recognised by the government as the Apex Tertiary Level Center for management of spinal injuries. However, the same facilities and expertise is not available in most other centers in the country [20], and thus most of the centers would not have been able to manage such a patient with the same kind of positive outcome. India does not have appropriate public health resources with only $3.93 \%$ of the GDP spent on health expenditure [21]. Rapid urbanization has occurred. However, $66 \%$ of the population still lives in rural areas [22], where health needs are mainly taken care by the public hospitals (primary health centers), which have only basic amenities and such patients could not have been appropriately managed. In fact, most of such centers would not have the multi-disciplinary team required for the management of such patients [20].

\section{Conclusion}

Acute traumatic spinal cord injury in a pregnant female patient in the third trimester is a difficult scenario with risks to both the mother and the fetus. With an interdisciplinary approach and vigilant protocol, surgical fixation of the spine can be performed under GA with good outcomes for both the mother and the fetus. Early initiation of rehabilitation and continuation of the pregnancy till term are the keys to successful outcomes.

\section{Compliance with ethical standards}

Conflict of interest The authors declare that they have no conflict of interest.

\section{References}

1. Popov I, Ngambu F, Mantel G, Rout C, Moodley J. Acute spinal cord injury in pregnancy: an illustrative case and literature review. J Obstet Gynaecol (Lahore). 2003;23:596-8.

2. Gençosmanoğlu BE, Hanci M, Yücesoy G, Madazli R, Yilmaz H, Özgen M. Spinal cord injury caused by gunshot wound during pregnancy. J Spinal Cord Med. 2001;24:123-6.

3. Vijay K, Shetty AP, Rajasekaran S. Symptomatic vertebral hemangioma in pregnancy treated antepartum. A case report with review of literature. Eur Spine J. 2008;17((SUPPL.2):S299-303.

4. Chilkoti GT, Mohta M, Duggal S, Saxena AK. Anaesthetic concerns of a pregnant patient with Pott's spine for spine surgery in prone position. Indian J Anaesth. 2016;60:518-9. p

5. Kaul R, Chhabra HS, Kanagaraju V, Mahajan R, Tandon V, Nanda A, et al. Antepartum surgical management of Pott's paraplegia along with maintenance of pregnancy during second trimester. Eur Spine J. 2016;25:1064-9.

6. Badve SA, Ghate SD, Badve MS, Rustagi T, MacChiwala T, Parekh AN, et al. Tuberculosis of spine with neurological deficit in advanced pregnancy: a report of three cases. Spine J. 2011;11: e9-16.

7. Walton NKD, Melachuri VK. Anaesthesia for non-obstetric surgery during pregnancy. Contin Educ Anaesth Crit Care Pain. 2006;6:83-5.

8. Upadya M, Saneesh PJ. Anaesthesia for non-obstetric surgery during pregnancy. Indian J Anaesth. 2016;60:234-41.

9. Nas K, Yazmalar L, Sah V, Aydın A, Öneş K. Rehabilitation of spinal cord injuries. World J Orthop. 2015;6:8-16.

10. Dvorak MF, Fisher CG, Fehlings MG, Rampersaud YR, Öner FC, Aarabi B, et al. The surgical approach to subaxial cervical spine injuries: An evidence-based algorithm based on the SLIC classification system. Spine (Phila Pa 1976). 2007;32:2620-9.

11. James AH. Pregnancy-associated thrombosis. Hematol Am Soc Hematol Educ Progr. 2009;2009:277-85.

12. Greer IA, Nelson-Piercy C. Low-molecular-weight heparins for thromboprophylaxis and treatment of venous thromboembolism in pregnancy: a systematic review of safety and efficacy. Blood Am Soc Hematol. 2005;106:401-7. p

13. Mccollough CH, Schueler BA, Atwell TD, Braun NN, Regner DM, Brown DL, et al. Radiation exposure and pregnancy: when should we be concerned? Radiographics. 2007;27:909-18.

14. Giorgino FL, Egan CG. Use of isoxsuprine hydrochloride as a tocolytic agent in the treatment of preterm labour: a systematic review of previous literature. Arzneimittelforschung. 2010;60:415-20.

15. Brownfoot FC, Gagliardi DI, Bain E, Middleton P, Crowther CA. Different corticosteroids and regimens for accelerating fetal lung maturation for women at risk of preterm birth. Cochrane Database Syst Rev. 2013;29:CD006764

16. Marks JD, Schreiber MD. Inhaled nitric oxide and neuroprotection in preterm infants. Clin Perinatol NIH Public Access. 2008;35:793-807.

17. Hocaloski S, Elliott S, Hodge K, McBride K, Hamilton L, McBride CB, et al. Perinatal care for women with spinal cord injuries: a collaborative workshop for consensus on care in Canada. Top Spinal Cord Inj Rehabil. 2017;23:386-96. 
18. Macejko AM, Schaeffer AJ. Asymptomatic bacteriuria and symptomatic urinary tract infections during pregnancy. Urol Clin North Am. 2007;34:35-42.

19. Dawood R, Altanis E, Ribes-Pastor P, Ashworth F. Pregnancy and spinal cord injury. Obstet Gynaecol. 2014;16:99-107.

20. Chhabra HS, Sharma S, Arora M. Challenges in comprehensive management of spinal cord injury in India and in the Asian Spinal Cord network region: findings of a survey of experts, patients and consumers. Spinal Cord. 2018;56:71-7.
21. Che. Health financing profile 2017 India Context: Macroeconomic situation GGHE-D+External resources PVT-D CHE. 2000. Available from: https://data.worldbank.org/indicator. Date accessed: 2018-09-30.

22. The World Bank. Rural population (\% of total population) I Data [Internet]. World Bank staff estimates based on the United Nations Population Division's World Urbanization Prospects: 2018 Revision. 2018 [cited 2018 Sep 30]. Available from: https://data.worldba nk.org/indicator/SP.RUR.TOTL.ZS. Date accessed: 2018-09-30. 